\title{
CATÁLOGO BIBLIOGRÁFICO DE LOS GASTERÓPODOS TERRESTRES DE LA COMUNIDAD DE MADRID
}

\author{
M. ${ }^{\mathrm{a}}$ T. Aparicio*
}

\begin{abstract}
RESUMEN
En la Comunidad de Madrid se han recolectado y posteriormente se han citado en la bibliografía un total de 47 especies de Gasterópodos terrestres pertenecientes a 18 familias de la subclase Pulmonada. Las familias con mayor número de especies citadas son tres Zonitidae, Higromiidae y Helicidae con seis, trece y seis especies respectivamente. Desde el punto de vista malacológico esta región se puede considerar como un área de transición en la que confluyen factores atlánticos y mediterráneos.

Palabras clave: Mollusca, Pulmonata, distibución geográfica, Comunidad de Madrid, España.
\end{abstract}

\begin{abstract}
Bibliographic catalogue of Terrestrial Gastropods from Comunidad de Madrid

A total of 47 terrestrial gastropod species belonging to 18 families of the subclass Pulmonata have been collected in Comunidad de Madrid and cited in the bibliography. Three families show the largest number of cited species: Zonitidae, Higromiidae and Helicidae with six, thirteen and six species respectively. From a malacological point of view this region may be considered as a transitional area in which atlantic and mediterranean factors converge.
\end{abstract}

Key words: Mollusca, Pulmonata, geographic distribution, Comunidad de Madrid, Spain.

\section{Introducción}

La bibliografía sobre los moluscos gasterópodos terrestres de la Comunidad de Madrid es poco abundante, lo cual resulta sorprendente en primer lugar por la importancia de esta comunidad en el centro de la Península Ibérica, y en segundo lugar por el número de especies citadas (47), bastante alto en relación al tamaño de la zona considerada. Se deben destacar los trabajos de Agüera et al.
(1992) y Parejo et al. (1993) como los trabajos mas recientes que realizan una recopilación crítica de los antecedentes bibliográficos previos de la Comunidad de Madrid, así como aportan los valiosos resultados de sus propios muestreos. En relación con grupos faunísticos mas concretos los trabajos de Tesis Doctoral de Prieto (1986), Altonaga (1988), Gómez (1988) y Puente (1994) recogen citas muy interesantes de los moluscos de nuestra comunidad. 
La escasez de estudios sobre la fauna malacológica terrestre de la comunidad de Madrid podría justificarse en parte por una previsible pobreza de este tipo de fauna en el área que estamos considerando como consecuencia de una mayoritaria existencia de suelos pobres en calcio y de un marcado clima continental con inviernos largos y fríos y veranos muy calidos secos y con alto grado de insolación, factores todos ellos que no favorecen este tipo de fauna (Sacchi, 1957, 1965) (Aparicio, 1982, 1986).

En el presente trabajo hemos llevado a cabo una actualización de los datos disponibles sobre la fauna malacológica la región considerada con las consecuencias que de ella se derivan.

\section{Resultados}

Se indican a continuación las 18 familias de la subclase Pulmonata a las que pertenecen las 47 especies que han sido citadas de la Comunidad de Madrid con la referencia bibliográfica de la que cada cita ha sido tomada. Esta bibliografía recoge también otras citas previas a las que no hacemos mención expresa para no alargar el trabajo.

\section{Familia COCHLICOPIDAE}

1.- Cochlicopa lubrica (Müller, 1774)

Gómez (1988); Parejo et al. (1993)

Familia PUPILLIDAE

2.- Lauria cylindracea (Da Costa, 1778)

Gómez (1988); Parejo et al. (1993)

\section{Familia VALLONIIDAE}

3.- Vallonia costata (Müller, 1774)

Gómez (1988); Agüera et al. (1992); Parejo et al. (1993)

4.- Vallonia pulchela (Müller, 1774)

Agüera et al. (1992)

5.- Vallonia excentrica (Sterki, 1892)

Parejo et al. (1993)

Familia VerTiginIDAE

6.- Truncatellina callicatris (Scacchi, 1833)

Gómez (1988); Agüera et al. (1992); Parejo et al. (1993)
7.- Vertigo antivertigo (Draparnaud, 1801) Gómez (1988)

\section{Familia CHONDRINIDAE}

8.- Granopupa granum (Draparnaud, 1801)

Gómez (1988); Agüera et al. (1992); Parejo et al. (1993)

\section{Familia BULIMINIDAE}

9.- Jaminia quadridens (Müller, 1774)

Gómez (1988); Agüera et al. (1992); Parejo et al. (1993)

10.- Ena obscura (Müller, 1774)

Gómez (1988); Parejo et al. (1993)

Familia FerussacidDae

11.- Ferussacia folliculus (Gmelin, 1790)

Parejo et al. (1993)

12.- Cecilioides acicula (Müller, 1774)

Agüera et al. (1992); Parejo et al. (1993)

Familia SUBULINIDAE

13.- Rumina decollata (Linneo, 1758)

Gorrotxategi et al. (1990); Agüera et al. (1992); Parejo et al. (1993)

\section{Familia Punctidae}

14.- Toltecia pusilla (Lowe, 1831) Agüera et al. (1992)

\section{Familia EUCONULIDAE}

15.- Euconulus fulvus (O.F. Müller, 1774) Altonaga (1988)

Familia VITRINIDAE

16.- Vitrina pellucida (O.F. Müller, 1774) Altonaga (1988)

17.- Phenacolimax annularis (Studer, 1820) Altonaga (1988)

\section{Familia ZoNITIDAE}

18.- Aegopinella nitidula (Draparnaud sensu Rossmaessler 1835)

Altonaga (1988) 
19.- Nasovitrea hammonis (Ström, 1765) Altonaga (1988)

20.- Vitrea crystallina (Müller, 1774) Parejo et al. (1993)

21.- Vitrea contracta (Westerlund, 1871) Agüera et al. (1992); Parejo et al. (1993)

22.- Oxychilus draparnaudi (Beck, 1837) Altonaga, (1988); Agüera et al. (1992); Parejo et al. (1993)

23.- Oxychilus cellarius (Müller, 1774)

Altonaga (1988); Parejo et al. (1993)

Familia ARIONIDAE

24.- Arion sp.

Agüera et al. (1992)

Familia MILACIDAE

25.- Milax gagates (Draparnaud, 1801) Agüera et al. (1992)

Familia LiMACIDAE

26.- Limax flavus (Linneo, 1758) Agüera et al. (1992)

27.- Lehmannia valentiana (Férussac, 1823) Agüera et al. (1992)

Familia AgriolimacidAE

28.- Deroceras reticulatum (Müller, 1774) Agüera et al. (1992)

\section{Familia HYGROMIIDAE}

29.- Monacha cartusiana (Müller, 1774) Aparicio (1983); Prieto (1986); Agüera et al. (1992); Parejo et al. (1993); Puente (1994)

30- Cochlicella barbara (Linneo, 1758) Aparicio (1983); Prieto (1986), Agüera et al. (1992); Parejo et al. (1993), Puente (1994)

31.- Oestophora barbula (Rossmassler, 1838) Prieto (1986); Puente (1994); Puente (1996)

32.- Candidula gigaxii (Pfeiffer, 1850) Aparicio (1983); Parejo et al. (1993); Puente (1994)

33.- Candidula intersecta (Poiret, 1801) ¿? Prieto (1986); Puente (1994)
34.- Helicella conspurcata (Draparnaud, 1801)

Agüera et al. (1992); Parejo et al. (1993); Puente y Altonaga (1995)

35.- Helicella itala (Linneo, 1758) Aparicio (1980); Aparicio (1986); Puente (1994)

36.- Helicella madritensis (Rambur, 1868) Prieto (1986); Puente (1994)

37.- Cernuella virgata (Da Costa, 1778) Aparicio (1983); Prieto (1986); Agüera et al. (1992); Parejo et al. (1993); Puente (1994)

38.- Microxeromagna armillata (Lowe, 1852)

Aparicio (1983); Agüera et al. (1992); Parejo et al. (1993); Puente (1994); Puente y Altonaga (1995)

39.- Xerosecta cespitum (Draparnaud, 1801)

Aparicio (1983); Prieto (1986); Agüera et al. (1992); Parejo et al. (1993); Puente (1994)

40.- Xerosecta reboudiana (Bourguignat, 1864) Aparicio (1983); Parejo et al. (1993); Puente (1994)

41.- Trochoidea geyeri (Soos, 1926)

Parejo et al. (1993)

\section{Familia HeLICIDAE}

42.- Cepaea nemoralis (Linneo, 1758)

Aparicio (1986); Prieto (1986); Parejo et al. (1993); Puente (1994)

43.- Eobania vermiculata (Müller, 1774) ¿?

Puente (1994)

44.- Helix aspersa (Müller, 1774)

Aparicio (1986); Prieto (1986); Agüera et al. (1992);

Parejo et al. (1993); Puente (1994)

45.- Iberus gualterianus (Linneo, 1758)

Puente (1994)

46.- Theba pisana (Müller, 1774)

Aparicio (1983); Prieto (1986); Agüera et al. (1992); Parejo et al. (1993); Puente (1994)

47.- Otala punctata (Müller, 1774)

Agüera et al. (1992)

\section{Discusión}

Las especies mas frecuentes y abundantes en la Comunidad de Madrid, de acuerdo con la bibliografía consultada, son Xerosecta cespitum, Microxeromagna armillata y Cernuella virgata. Estas especies presentan una distribución medite- 
rránea y viven en biotopos expuestos y ruderalizados (Kerney et al., 1983), que están muy extendidos ambos en la zona considerada. Quizá la relativa abundancia de este tipo de biotopos, expuestos y ruderales, en nuestra comunidad, posibilita la existencia de una fauna malacológica que de otro modo sería difícil encontrar en una región con las condiciones litográficas, climáticas y de vegetación como la que nos ocupa (Sacchi y Nos, 1965).

La mayoría de las especies halladas en esta región son de distribución mediterránea, como cabía esperar por las condiciones climáticas y de vegetación predominantes en la Comunidad de Madrid. No obstante una pequeña proporción de las especies aquí mencionadas son de distribución Holártica y del Oeste de Europa, solapando en esta región su distribución con las especies marcadamente mediterraneas. Cabe mencionar, en este sentido el Escorial y el valle del río Lozoya, que se presentan como reductos para la presencia de algunas especies características de regiones mas meridionales (Altonaga 1988; Puente, 1994). Así por ejemplo, Euconulus fulvus aunque es una especie ubiquista de distribución holartica, ha sido recogida en el Escorial (Altonaga, 1988); Vitrina pellucida también de distribución holártica ha sido hallada también en el Escorial, en Cotos y en Robledondo; Phenacolimax anularis especie montana del centro de Europa se ha citado del Escorial, el Pardo y el Paular. Aegopinella nitidula especie europea subatlantica se ha encontrado en el valle del Lozoya siendo el sistema central su límite sur (Altonaga, 1988). Nasovitrea hammonis especie paleartica, se ha citado de Robledondo; Oxichilus cellarius mas ubiquista aunque europea occidental, del Paular. Otros dos ejemplos de las familias Higromiidae y Helicidae respectivamente serían Helicilla itala y Cepaea nemoralis, que aunque algo mas abundantes en la zona considerada que las especies que acabamos de mencionar, presentan una distribución típicamente oeste europea, donde la región central puede considerarse probablemente una de sus zonas limítrofes sur de distribución.

Esta actualización sobre la fauna malacológica de la Comunidad de Madrid confirma de nuevo la consideración de esta zona como un área de transición que aunque con una mayoría de elementos mediterráneos presenta en su parte norte algunas especies europeas con alguna influencia atlántica (Aparicio, 1986; Agüera et al., 1992; Parejo et al., 1993).

\section{AGRADECIMIENTOS}

Este trabajo ha sido financiado por el Proyecto de Investigación de la Comunidad de Madrid 2004/AMB/0750.

\section{Referencias}

Agüera, L., Parejo, C. \& Muñoz, B., 1992. Fauna malacológica terrestre de la vega de Aranjuez y laguna de Ontígola. Graellsia, 48: 25-33.

Altonaga, K., 1988. Estudio taxonómico y biogeográfico de las Familias Endodontidae, Euconulidae, Zonitidae y Vitrinidae (Gastropoda: Pulmonata: Stylommatophora) de la Península Ibérica, con especial referencia al País Vasco y zonas adyacentes. Tesis Doctoral, Universidad del País Vasco. 549 pp.

ApARICIO, M. T., 1980. Variaciones en la concha de Helicella (Helicella) itala (Gastropoda: Pulmonata) en la región del alto Tajo. Comunicaciones del Primer Congreso Nacional de Malacología, Madrid: 35-40.

APARICIO, M. T., 1982. Observations on the anatomy of some Helicidae from central Spain. Malacologia, 22: 621-626.

APARICIO, M. T., 1983. Estudio morfológico y citotaxonómico de algunos Helicidos de la fauna española en especial de la región central. Tesis doctoral, 1981. Universidad Complutense de Madrid. 267 pp.

Aparicio, M. T., 1986. The geographic distribution of the family Helicidae in central Spain. Proceedings of the $8^{\text {th }}$ Internacional Malacological Congress, Budapest, 1983: 1-6.

Gómez, B., 1988. Estudio sistemático y biogeográfico de los moluscos terrestres del suborden Orthurethra (Gastropoda: Pulmonata: Stylommatophora) del País Vasco y regiones adyacentes y catalogo de las especies ibéricas. Tesis Doctoral, Universidad del País Vasco. 424 pp.

Gorrotxategi, R., Abalde, A., Altonaga, K., Angulo, E., Prieto, C. \& Puente, A., 1990. Nuevos datos sobre la distribución en la Península Ibérica de Rumina decollata (Linnaeus 1758) (Pulomonata: Stylommatophora: Subulinidae). Cuadernos de Investigación Biológica (Bilbao), 16: 115-124.

Kerney, M. P., Cameron, R. A. D. \& Jungbluth, J. H., 1983. Die Landschnecken Nord-und Mitteleuropas. Paul Parey. Hamburg und Berlin. 384 pp.

Parejo, C., Refoyo, P., Almodóvar, A. \& Muñoz, B., 1993. Contribución al conocimiento de la malacofauna terrestre entre los ríos Henares, Jarama y Tajuña en la Comunidad Autónoma de Madrid. Graellsia, 49: 77-85.

PRIETO, C., 1986. Estudio sistemático y biogeográfico de los Helicidae sensu Zilch, 1959-60 (Gastropoda: Pulmonada: Stylommatophora) del País Vasco y regiones adyacentes. Tesis Doctoral, Universidad del País Vasco. 393 pp. + 10 lám.

Puente, A., 1994. Estudio taxonómico y biogeográfico de la superfamilia Helicoidea Rafinesque, 1815 (Gastropoda: Pulmonata: Stylommatophora) de la Península Ibérica e islas Baleares. Tesis Doctoral, Universidad del País Vasco. 970 pp. 
Puente, A. \& Altonaga, K., 1995. Estudio morfológico y corológico de dos especies conquiológicamente similares, Helicilla conspurcata (Draparnaud, 1801) y Microxeromagna armillata (Lowe, 1852), en la Península Ibérica e islas Baleares (Pulmonada: Helicoidea: Hygromiidae). Zoologica Baetica, 6: 121-148.

SACCHI, C. F., 1957. Lineamenti biogeografici della Spagna mediterranea su basi malacofaunistiche. Publicaciones del Instituto de Biología Aplicada, 25: 5-48.
SACCHI, C. F., 1965. Ecological and historical bases for a study of the Iberian terrestrial mollusca. Proceedings of the First European Malacological Congress: 243-257.

SACCHI, C. F. \& Nos, R., 1958. Quelques distributions interessantes des Mollusques terrestres ibériques. Publicaciones del Instituto de Biología Aplicada, 27: 89-95. 